Pacific Journal of Mathematics

HELICOID OF CONSTANT MEAN CURVATURE AND THEIR
GAUSS MAPS 


\title{
HELICOIDS OF CONSTANT MEAN CURVATURE AND THEIR GAUSS MAPS
}

\author{
WALTER SEAMaN
}

\begin{abstract}
A helicoidal surface in $\mathbf{R}^{3}$ is a natural generalization of a surface of revolution. We give a simple description via the theory of harmonic maps of the Gauss maps and Gaussian images of complete helicoidal surfaces of constant mean curvature in $\mathbf{R}^{3}$. Do Carmo had conjectured that the Gaussian image of such a surface contained an equator. This is true for the complete surfaces of revolution of constant mean curvatures in $\mathbf{R}^{3}$ and we affirm this for helicoids of constant mean curvature.
\end{abstract}

In fact, there is such a surface of revolution (an "unduloid", see §1) whose Gaussian image lies in an arbitrarily thin symmetric band about an equator. We show that this is also the case for the complete helicoids of constant mean curvature, which affirms the do Carmo conjecture. And, whereas the Gauss map for a surface of revolution of constant mean curvature is controlled by the pendulum equation, we show that the Gauss map for a helicoidal surface is governed by a pendulum equation "with infinite restoring force". We also compute a one-parameter family of such Gauss maps corresponding to the associate family ([5]) of the helicoid. This gives a one-parameter family of harmonic (non \pm holomorphic) maps into $S^{2}$ (see §3), which includes the pendulum-type as a particular member. Previously, only the pendulum-type seems to have been well known.

Our original interest in this topic came from the recent work of Hoffman, Osserman, and Schoen ([3]). They proved that the Gaussian image of a complete surface of constant mean curvature in $\mathbf{R}^{3}$ cannot lie in a closed hemisphere unless it is a plane or a right circular cylinder. This result leads to the question: what can the Gaussian image of a complete surface of constant mean curvature look like? It is this question to which we address ourselves in this paper, at least for the special case of helicoidal surfaces.

1. We recall some well-known facts from surface theory. Suppose a surface of revolution in $\mathbf{R}^{3}$ is given locally by

$$
f(r, t)=(c(r) \cos t, c(r) \sin t, r), \quad c(r) \neq 0,
$$

$(r, t)$ in some domain $D$. 
The mean curvature function for $f$ is given by

$$
H=\frac{1+\dot{c}^{2}-c \ddot{c}}{2 c\left(1+\dot{c}^{2}\right)^{3 / 2}} .
$$

For $H$ constant, a first integral of (1.1) is given by

$$
0=-H c^{2}+c / \sqrt{1+\dot{c}^{2}} \mp k^{2}
$$

where

$$
\pm k^{2}=c_{1}(0) / \sqrt{1+\dot{c}(0)^{2}}-H c_{1}^{2}(0)
$$

or, equivalently,

$$
0=c^{2}+2 a c / \sqrt{1+\dot{c}^{2}} \mp b^{2} .
$$

Delaunay [1] first realized that the solutions to this equation are roulettes: the solution is the roulette of an ellipse if we take $+b^{2}$, the roulette of a hyperbola if we take $-b^{2}$.

Note that in case $b=0$, we get either that $c(r)$ is constant, in which case $f$ is a right circular cylinder, or $(r, c(r))$ is a circle, in which case $f$ is a sphere.

If we let $r(s)$ be the solution to

$$
r(s)=\int \frac{c(r(s)) d s}{\sqrt{1+\left(\frac{d c}{d r}(r(s))\right)^{2}}},
$$

then the coordinates $(s, t)$ are isothermal for $f$.

The Gauss map for $f$ in $(s, t)$ coordinates is given by

$$
(s, t) \rightarrow\left(e^{i t} \sin \alpha(s), \cos \alpha(s)\right),
$$

where

$$
\begin{aligned}
& \sin \alpha(s)=\frac{-1}{\sqrt{1+\left(\frac{d c}{d r}(r(s))\right)^{2}}} \\
& \cos \alpha(s)=\frac{\frac{d c}{d r}(r(s))}{\sqrt{1+\left(\frac{d c}{d r}(r(s))\right)^{2}}}
\end{aligned}
$$


By direct calculation, using (1.2), one verifies that

$$
\dot{\alpha}^{2}=\sin ^{2} \alpha \pm b^{2} / a^{2}
$$

where $-b^{2} / a^{2}$ corresponds to the roulette of the ellipse, or "undulary", whose surface of revolution is called an "unduloid", while $+b^{2} / a^{2}$ corresponds to the roulette of the hyperbola, or "nodary", whose surface of revolution is called a "nodoid". Actually, (1.4) is just the first integral to the system

$$
\ddot{\alpha}=\frac{\sin 2 \alpha}{2}, \quad \pm \frac{b^{2}}{a^{2}}=\dot{\alpha}_{0}^{2}-\sin ^{2} \alpha_{0}, \quad \alpha(0)=\alpha_{0}, \dot{\alpha}(0)=\dot{\alpha}_{0},
$$

which is the pendulum equation, shifted by $\pi / 2$ (i.e., substitute $\alpha(-\pi / 2)$ for $\alpha$ ).

The trivial solution to (1.5): $\alpha \equiv n \pi / 2$, corresponds to the right circular cylinder if $n$ is odd, and to the plane if $n$ is even. The solutions $\alpha=($ odd $) \pi / 2$ are stable, and this yields the well-known result that the Gaussian image of an unduloid can be made an arbitrarily thin symmetric band about an equator. This is because if $\left(\alpha_{0}, \dot{\alpha}_{0}\right)$ is near $(\pi / 2,0)$ then $\dot{\alpha}_{0}^{2}-\sin ^{2} \alpha_{0}$ is negative, hence we get $-b^{2} / a^{2}$ in (4), which corresponds to the unduloid. The critical value $\dot{\alpha}_{0}^{2}=\sin ^{2} \alpha_{0}$, i.e., $b=0$, corresponds to the sphere (unless $\alpha_{0} \equiv 0$, say), while the nodoids correspond to $\dot{\alpha}_{0}^{2}>$ $\sin ^{2} \alpha_{0}$. In each case, the Gaussian image can be read off of the phase portrait for the pendulum equation.

2. Consider the one-parameter group $g_{t}$ of rigid motions on $\mathbf{R}^{3}$ given by

$$
g_{t}:(x, y, z) \rightarrow(x \cos t+y \sin t,-x \sin t+y \cos t, z+h t), \quad t \in \mathbf{R} .
$$

$g_{t}$ is called a helicoidal motion with axis $0 z$ and pitch $h$. A helicoidal surface with axis $0 z$ and pitch $h$ is a surface that is invariant under $g_{t}$ for all $t$. If $h=0$, this is just a surface of revolution. Let $M$ be such a surface. Let $U$ be an open set in $M$ such that the intersection of $U$ with some plane $\Pi$ containing $0 z$ is a curve given by $z=\lambda\left(\sqrt{x^{2}+y^{2}}\right)$. Then we can parameterize $U$ by

$$
(r, \theta) \rightarrow(r \cos \theta, r \sin \theta, \lambda(r)+h \theta)
$$

where $(r, \theta)$ are polar coordinates in the $x y$-plane. A nicer parameterization $(s, t)$ for such a surface would be achieved by letting the $s$-curves be the trajectories of the helicoidal motion (i.e., helices), parameterized by arclength, and letting the $t$-curves be their orthogonal trajectories. This 
"natural" parameterization is exhibited in [2], and is given by

$$
(s, t) \rightarrow(\rho(s) \cos \phi(s, t), \rho(s) \sin \phi(s, t), \lambda(s)+h \phi(s, t)),
$$

where $\phi_{t t}=0$.

The metric in these coordinates is given by

$$
d \sigma^{2}=d s^{2}+U^{2}(s) d t^{2},
$$

where $U^{2}(s)=\left(\rho^{2}(s)+h^{2}\right) / m^{2}, m$ a constant.

This parameterization is well defined for all $s$ such that $\rho(s) \neq 0$ (see [2]).

We see that the Gauss map for a helicoid can be written

$$
(s, t) \rightarrow \frac{1}{\left(\dot{\lambda}^{2} \rho^{2}+\dot{\rho}^{2}\left(\rho^{2}+h^{2}\right)^{1 / 2}\right.}\left(\dot{\lambda} \rho+i h \rho e^{l \phi}, \rho \dot{\rho}\right) .
$$

By collapsing terms, we see that this Gauss map can be written

$$
(s, t) \stackrel{X}{\rightarrow}\left(e^{i \beta(s, t)} \sin \alpha(s), \cos \alpha(s)\right), \quad \text { with } \beta_{t t}=0 .
$$

If we define $\bar{s}(s)=\int_{0}^{s} U^{-1}(s) d s$, then it's easy to check that the parameters $(\bar{s}, t)$ are isothermal, and relabelling $\bar{s}$ by $s$, we see that the Gauss map of any helicoidal surface can be written, in isothermal parameters, by (2.1). Of course, it may well be that there are non-helicoidal surfaces with Gauss maps of the form (2.1)

We are interested in studying the Gauss map and Gaussian image of a helicoid of constant mean curvature. It is well known that a surface in $\mathbf{R}^{3}$ has constant mean curvature if and only if its Gauss map is a harmonic map. This means that if we have isothermal parameters on the surface, then the Gauss map, considered as a map from the surface into $\mathbf{R}^{3}$, satisfies

$$
\Delta X+|\Delta X|^{2} X=0 .
$$

Furthermore, (2.2) shows that the harmonicity of $X$ is independent of the conformal structure of the surface, so that (2.2) can be computed with $X$ as a map from the plane with the standard flat metric into $\mathbf{R}^{3}$. Finally, Kenmotsu [3] has shown that given any harmonic, nowhere \pm holomorphic map $f$ of a Riemann surface $\delta$ into $S^{2}$, there is a surface of constant mean curvature $H(\neq 0)$ in $\mathbf{R}^{3}$, unique up to translation, which has Gauss map, in stereographic coordinates, $f$. The metric on the surface is given by

$$
\left[\frac{2}{H} \frac{\left|f_{\bar{z}}\right|}{1+|f|^{2}}\right]^{2}|d z|^{2}
$$

where $z$ is a conformal parameter on $\delta$. 
The harmonicity equation (2.2) for the map

$$
X:(s, t)=\left(e^{i \beta(s, t)} \sin \alpha(s), \cos \alpha(s)\right)
$$

yields the system of equations:

$$
\begin{gathered}
\ddot{\alpha}=|\nabla \beta|^{2} \frac{\sin 2 \alpha}{2}, \\
2 \dot{\alpha} \dot{\beta} \cos \alpha+\Delta \beta \sin \alpha=0
\end{gathered}
$$

where dot denotes differentiation with respect to $s$. Let us make the "helicoidal" assumption that $\beta_{t t}=0$. Thus $\beta(s, t)=b(s)+c(s) t$, but since (2.3) implies that $|\nabla \beta|^{2}$ is independent of $t$, we must have

$$
\beta(s, t)=b(s)+c t .
$$

Now assume that $\alpha \in(0, \pi)$. Then (2.3), (2.4) can be written as the system:

$$
\left(\begin{array}{c}
\dot{\alpha} \\
\dot{\alpha} \\
\dot{b}
\end{array}\right)=\left(\begin{array}{c}
\dot{\alpha} \\
\left(\dot{b}^{2}+c^{2}\right) \frac{\sin ^{2} \alpha}{2} \\
-2 \dot{\alpha} \dot{b} \cot \alpha
\end{array}\right) .
$$

This system has a unique local solution for each given $\left(\alpha_{0}, \dot{\alpha}_{0}, \dot{b}_{0}\right) \in$ $(0, \pi) \times \mathbf{R}^{2}$. In particular, if $\dot{b}_{0}=0$, then the solution to $(2.5)$ is given by the solution to

$$
\left(\begin{array}{c}
\alpha \\
\dot{\alpha}
\end{array}\right)=\left(\begin{array}{c}
\dot{\alpha} \\
c^{2} \frac{\sin 2 \alpha}{2}
\end{array}\right)
$$

(the pendulum equation) $\alpha(0)=\alpha_{0}, \dot{\alpha}(0)=\dot{\alpha}_{0}, \dot{b} \equiv 0$, i.e., if $b_{0}=0$, then $\dot{b} \equiv 0$. This case corresponds to the Gauss map of a surface of revolution, which is well understood (see $\S 1$ ). Therefore, we will assume $\dot{b_{0}} \neq 0$ and hence $\dot{b}$ is never 0 .

In this case we can divide by $\dot{b}$ in (2.4) and after an elementary integration, deduce that

$$
\beta(s, t)=\dot{b}_{0} \sin ^{2} \alpha_{0} \int_{0}^{s} \csc ^{2} \alpha(s) d s+c t .
$$

Using (2.7), (2.3) becomes

$$
\ddot{\alpha}=\left(\dot{b}_{0}^{2} \sin ^{4} \alpha_{0} \csc ^{4} \alpha+c^{2}\right) \frac{\sin 2 \alpha}{2},
$$

with initial conditions $\alpha(0)=\alpha_{0}, \dot{\alpha}(0)=\dot{\alpha}_{0}$. 
A first integral of (2.8) is given by

$$
\alpha^{2}=\dot{\alpha}_{0}^{2}+\left(\dot{b}_{0}^{2}-c^{2}\right) \sin ^{2} \alpha_{0}-\dot{b}_{0}^{2} \csc ^{2} \alpha+c^{2} \sin ^{2} \alpha .
$$

A phase portrait analysis of (2.9) shows that the solutions to (2.8) are globally defined and periodic, i.e, the trajectories given by (2.9) are closed and symmetric about $(\pi / 2,0)$. The assumption that $\dot{b}_{0} \neq 0$ is crucial here. Solutions to equation (2.8) are described "physically" by a pendulum with infinite restoring force, since, no matter how big the initial force $\left(\dot{\alpha}_{0}\right)$ we impart to the pendulum, it always swings back and varies periodically about the equilibrium $\left(\alpha_{0}, \dot{\alpha}_{0}\right)=(\pi / 2,0)$. Finally, it is elementary to show that the trivial solution $\alpha \equiv \pi / 2$ is stable.

We now see that $X$ is defined as a map from all of $\mathbf{R}^{2}$ into $S^{2}$, and its image is a symmetric band about the equator in the " $x y$ " plane. This band can be made arbitrarily thin owing to the aforementioned stability result.

Let us now normalize the class of all such maps $X$ by assuming that $\alpha_{0}=\pi / 2$, which simply amounts to adjusting $\dot{b}_{0}$, since every solution to (2.8) must pass through $\pi / 2$ at some $s$. If we let $f=\sigma \circ X$, where $\sigma=$ the stereographic projection from the north pole of $S^{2}$ in $\mathbf{R}^{3}$, then we have

$$
f=\frac{e^{i \beta} \sin \alpha}{1-\cos \alpha}
$$

by direct verification; if $\dot{b}_{0} \neq 0$, then, for $z=s+i t, f_{z}, f_{\bar{z}} \neq 0$, and $X$ determines a unique (up to translation) surface $S_{X}$ of constant mean curvature $H$ in $\mathbf{R}^{3}$, whose Gauss map in stereographic coordinates is $f$.

The metric of this surface (i.e., the metric induced on $\mathbf{R}^{2}$ via $X$ ) is given by

$$
\left[\frac{2\left|f_{\bar{z}}\right|}{H\left(1+|f|^{2}\right)}\right]^{2}|d z|^{2} \text {. }
$$

We can assume $H=1 / 2$, and the metric then becomes

$$
\left(\dot{b}_{0}^{2} \csc ^{2} \alpha+(\dot{\alpha}+c \sin \alpha)^{2}\right)|d z|^{2}:=F(s)|d z|^{2} .
$$

Note that since $\alpha$ is periodic, we have $F(s) \geq \varepsilon>0 \forall s$, and since $F(s)$ is defined for all $s$, the metric (2.10) is complete. This says that, in particular, the Gaussian image of a complete helicoidal surface of constant mean curvature is a symmetric band about an equator, and, if the surface is either an unduloid, or else not a surface of revolution, then this band can be made arbitrarily thin. 
3. The question still remains: what are the surfaces of $S_{X}$ ? In particular, are there any non-helicoidal ones? We show that, unfortunately, each $S_{X}$ is a helicoid by showing that each $S_{X}$ is associate to a surface of revolution. The idea behind this is the following: if in (2.8), we simply allow $\dot{b_{0}}$ to approach 0 , then the resulting Gauss map will approach the Gauss map for a surface of revolution. However, this surface of revolution will have no a priori metric relation to the starting surface $S_{X}$. What we want to do is let $\dot{b}_{0} \rightarrow 0$ and keep $F(s)|d z|^{2}$ constant, for this will show that in fact $S_{X}$ is isometric to a surface of revolution; hence $S_{X}$ is in the associate family to a surface of revolution (of constant mean curvature) and, therefore ([4], §13), must be helicoidal.

Our starting data is:

$$
\begin{aligned}
\ddot{\alpha} & =\left(\dot{b_{0}} \csc ^{4} \alpha+c^{2}\right) \frac{\sin 2 \alpha}{2} \\
\alpha(0) & =\pi / 2, \quad \dot{\alpha}(0)=\dot{\alpha}_{0} . \\
F(s) & =\left(\dot{b}_{0} \csc ^{2} \alpha+(\dot{\alpha}+c \sin \alpha)^{2}\right) .
\end{aligned}
$$

Using (2.9), one checks that

$$
\dot{F}(s)=2 c \cos \alpha F(s),
$$

so that

$$
\begin{aligned}
F(s) & =F(0) e^{\int_{0}^{s} 2 c \cos \alpha(s) d s} \\
& =\left(\dot{b}_{0}+\left(\dot{\alpha}_{0}+c\right)^{2}\right) e^{\int_{0}^{s} 2 c \cos \alpha(s) d s} .
\end{aligned}
$$

We want to vary the parameters $\dot{b}_{0}, \dot{\alpha}_{0}, c$ so that $\dot{b}_{0}$ will attain the value 0 , and $F(s)$ will remain constant. If we consider $\dot{b}_{0}, \dot{\alpha}_{0}, c$ as being functions of a variable, say $\theta$, then of course $\alpha$, and hence $F$, depends also on $\theta$, and what we are after are conditions on $\dot{b}_{0}(\theta), \dot{\alpha}_{0}(\theta), c(\theta)$ which will guarantee that $F$ does not depend on $\theta$. If we knew a priori that $F$ was differentiable as a function of $\theta$, then the condition that $F$ be independent of $\theta$ would simply be $\partial F / \partial \theta=0$, which would in turn be equivalent to

$$
\begin{gathered}
\dot{b_{0}}(\theta)+\left(\dot{\alpha}_{0}^{2}(\theta)+c(\theta)\right)^{2}=\text { constant } \\
\frac{\partial g}{\partial \theta}=0 \quad \text { where } g=2 c \cos \alpha .
\end{gathered}
$$

Now, using (2.8), one verifies that $g$ satisfies

$$
\frac{\partial^{2} g}{\partial s^{2}}=2 g\left(g^{2}-2\left(\dot{\alpha}_{0}(\theta)+\dot{b}_{0}^{2}(\theta)+c^{2}(\theta)\right)\right)
$$


and

$$
\begin{gathered}
g(0, \theta)=0 \\
\frac{\partial g(0, \theta)}{\partial s}=-2 c(\theta) \dot{\alpha}_{0}(\theta) .
\end{gathered}
$$

If we choose $c(\theta), \dot{\alpha}_{0}(\theta)$ and $\dot{b}_{0}(\theta)$ so that both $\dot{\alpha}_{0}(\theta) c(\theta)$ and $\dot{\alpha}_{0}^{2}(\theta)+$ $\dot{b}_{0}^{2}(\theta)+c^{2}(\theta)$ are constants, say $a$ and $d^{2}$, respectively, then (3.2) is satisfied. In this case, $g$ satisfies

$$
\begin{gathered}
\frac{\partial^{2} g}{\partial s^{2}}=2 g\left(g^{2}-2 d^{2}\right), \\
g(0, \theta)=0
\end{gathered}
$$

and

$$
\frac{\partial g}{\partial s}(0, \theta)=-2 a
$$

The point now is that $\left(3.4^{\prime}\right)$ has a unique local solution satisfying (3.5') (3.6') and $\partial g(0, \theta) / \partial \theta=0$, since the curve $(0, \theta)$ is non-characteristic. Furthermore, since all the data are analytic, we see that the solution to $\left(3.4^{\prime}\right)-\left(3.6^{\prime}\right)$, and $\partial g(s, \theta) / \partial \theta=0 \forall s$, is obtained simply by solving $\left(3.4^{\prime}\right)$ as an ODE in $s$, with initial conditions $g(0)=0, \dot{g}(0)=-2 a$. This $g$ then solves the problem $\partial F / \partial \theta=0$.

Consider our original $\dot{b}_{0}, \dot{\alpha}_{0}, c$ simply as constants, and define

$$
\dot{b_{0}}(\theta)=\sqrt{\left(\dot{\alpha}_{0}^{2}+\dot{b}_{0}^{2}+c^{2}\right)} \sin \theta,
$$

(3.8) $c^{2}(\theta)=\frac{\left(\dot{\alpha}_{0}^{2}+\dot{b}_{0}^{2}+c^{2}\right) \cos ^{2} \theta \pm \sqrt{\left(\dot{\alpha}_{0}^{2}+\dot{b}_{0}^{2}+c^{2}\right)^{2} \cos ^{4} \theta-4 c^{2} \dot{\alpha}_{0}^{2}}}{2}$

where we take the + root if $\dot{\alpha}_{0}<c^{2}$, the - root if $\dot{\alpha}_{0}>c^{2}$, either root $\dot{\boldsymbol{\alpha}}_{0}^{2}=c^{2}$, and finally

$$
\dot{\alpha}_{0}(\theta)=\frac{c \dot{\alpha}_{0}}{c(\theta)}
$$

Note that $c(\theta) \neq 0$, and the discriminate in (3.8) is positive for $\theta$ in the interval $\left(-\theta_{1}, \theta_{1}\right)$ where

$$
\theta_{1}=\cos ^{-1} \sqrt{\frac{\left|2 c \dot{\alpha}_{0}\right|}{\dot{\alpha}_{0}^{2}+\dot{b}_{0}^{2}+c^{2}}} .
$$


Also, for

$$
\theta_{0}=\cos ^{-1} \sqrt{\frac{c^{2}+\dot{\alpha}_{0}^{2}}{c^{2}+\dot{\alpha}_{0}^{2}+\dot{b}_{0}^{2}}},
$$

we have $c\left(\theta_{0}\right)=c, \dot{\alpha}_{0}\left(\theta_{0}\right)=\dot{\alpha}_{0}, \dot{b}_{0}\left(\theta_{0}\right)=\dot{b}_{0}$.

Finally, a short calculation shows that

$$
c^{2}(\theta)+\dot{\alpha}_{0}^{2}(\theta)+\dot{b}_{0}^{2}(\theta) \equiv c^{2}+\dot{\alpha}_{0}^{2}+\dot{b}_{0}^{2} .
$$

(Notice that $c^{2}(\theta)$ is a solution to

$$
\begin{gathered}
c^{4}(\theta)-\left(\dot{\boldsymbol{\alpha}}^{2}+\dot{b}_{0}^{2}+c^{2}\right) \cos ^{2}(\theta) c^{2}(\theta)+c^{2} \dot{\boldsymbol{\alpha}}_{0}^{2}=0 \\
\Leftrightarrow \frac{c^{2} \dot{\boldsymbol{\alpha}}_{0}^{2}+c^{4}(\theta)}{c^{2}(\theta)}=\left(\dot{\boldsymbol{\alpha}}_{0}^{2}+\dot{b}_{0}^{2}+c^{2}\right) \cos ^{2} \theta \\
\left.\Leftrightarrow \dot{\boldsymbol{\alpha}}_{0}^{2}(\theta)+c^{2}(\theta)=\left(\dot{\boldsymbol{\alpha}}_{0}^{2}+\dot{b}_{0}^{2}+c^{2}\right) \cos ^{2} \theta .\right)
\end{gathered}
$$

Our metrics $F(s)|d z|^{2}$ are thus all independent of $\theta$, where $\theta$ corresponds to the one-parameter family of solutions:

$$
\begin{aligned}
& \ddot{\alpha}=\left(\left(\dot{\alpha}_{0}^{2}+\dot{b}_{0}^{2}+c^{2}\right) \sin ^{2} \theta \csc ^{4} \alpha\right. \\
& +\frac{\left.\left(\dot{\alpha}_{0}^{2}+\dot{b}_{0}^{2}+c^{2}\right) \cos ^{2} \theta \pm\left(\left(\dot{\alpha}_{0}^{2}+\dot{b}_{0}^{2}+c^{2}\right)^{2} \cos ^{4} \theta-4 c^{2} \dot{\alpha}_{0}^{2}\right)^{1 / 2}\right) \frac{\sin 2 \alpha}{2},}{\alpha(0)=\frac{\pi}{2},} \\
& \dot{\alpha}(0)=\frac{c \dot{\alpha}_{0}}{\frac{\left(\dot{\alpha}_{0}^{2}+\dot{b}_{0}^{2}+c^{2}\right) \cos ^{2} \theta \pm\left(\left(\dot{\alpha}_{0}^{2}+\dot{b}_{0}^{2}+c^{2}\right)^{2}-4 c^{2} \dot{\alpha}_{0}^{2}\right)^{1 / 2}}{2}}
\end{aligned}
$$

For $\theta=0$, we simply have

$$
\ddot{\alpha}=K \frac{\sin 2 \alpha}{2},
$$

i.e., the pendulum equation, which corresponds to a surface of revolution. Finally, this shows that $S_{X}$ is associate to a surface of revolution, and hence is helicoidal. 
The one-parameter family of harmonic maps is given by $(2.1),(2.7)$, and (2.8), where $\alpha_{0}=\pi / 2$, and $\dot{\alpha}_{0}, \dot{b}_{0}, c$ are replaced by $\dot{\alpha}_{0}(\theta), \dot{b_{0}}(\theta)$, $c(\theta)$, respectively.

\section{REFERENCES}

[1] C. Delaunay, Sur la surface de révolution dont la courbe moyenne est constante, J. Math. pures et appl., Sér. 1 (6) (1841), 309-320.

[2] M. do Carmo and M. Dajczer, Helicoidal surfaces with constant mean curvature, preprint.

[3] D. Hoffman, R. Osserman and R. Schoen, On the Gauss map of complete surfaces at constant mean curvature in $\mathbf{R}^{3}$ and $\mathbf{R}^{4}$, to appear.

[4] K. Kenmotsu, Weierstrass formula for surfaces of prescribed mean curvature, Math. Ann., 245 (1979), 89-99.

[5] B. Lawson, Complete minimal surfaces in $S^{3}$, Ann. of Math., 92 (1970), 335-374.

Received November 11, 1982.

STANFORD UNIVERSITY

STANFORD, CA 94305

Current address: University of Iowa

Iowa City, IA 52242 


\title{
PACIFIC JOURNAL OF MATHEMATICS EDITORS
}

\author{
DONALD BABBITT (Managing Editor) \\ University of California \\ Los Angeles, CA 90024 \\ Hugo Rossi \\ University of Utah \\ Salt Lake City, UT 84112 \\ C. C. MOORE and Arthur Ogus \\ University of California \\ Berkeley, CA 94720
}

\author{
J. Dugundir \\ Department of Mathematics \\ University of Southern California \\ Los Angeles, CA 90089-1113
}

R. FINN and H. SAMELSON

Stanford University

Stanford, CA 94305

ASSOCIATE EDITORS
R. ARENS
E. F. BECKENBACH
B. H. NeumanN
F. WOLF
K. YosHIDA (1906-1982)

\section{SUPPORTING INSTITUTIONS}

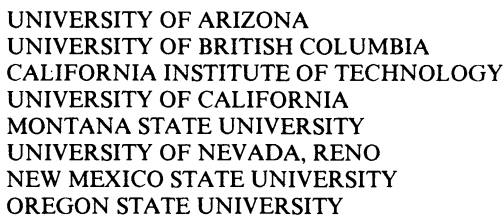

\author{
UNIVERSITY OF OREGON \\ UNIVERSITY OF SOUTHERN CALIFORNIA \\ STANFORD UNIVERSITY \\ UNIVERSITY OF HAWAII \\ UNIVERSITY OF TOKYO \\ UNIVERSITY OF UTAH \\ WASHINGTON STATE UNIVERSITY \\ UNIVERSITY OF WASHINGTON
}

The Supporting Institutions listed above contribute to the cost of publication of this Journal, but they are not owners or publishers and have no responsibility for its content or policies.

Mathematical papers intended for publication in the Pacific Journal of Mathematics should be in typed form or offset-reproduced (not dittoed), double spaced with large margins. Please do not use built up fractions in the text of the manuscript. However, you may use them in the displayed equations. Underline Greek letters in red, German in green, and script in blue. The first paragraph must be capable of being used separately as a synopsis of the entire paper. In particular it should contain no bibliographic references. Please propose a heading for the odd numbered pages of less than 35 characters. Manuscripts, in triplicate, may be sent to any one of the editors. Please classify according to the scheme of Math. Reviews, Index to Vol. 39. Supply name and address of author to whom proofs should be sent. All other communications should be addressed to the managing editor, or Elaine Barth, University of California, Los Angeles, California 90024.

There are page-charges associated with articles appearing in the Pacific Journal of Mathematics. These charges are expected to be paid by the author's University, Government Agency or Company. If the author or authors do not have access to such Institutional support these charges are waived. Single authors will receive 50 free reprints; joint authors will receive a total of 100 free reprints. Additional copies may be obtained at cost in multiples of 50 .

The Pacific Journal of Mathematics is issued monthly as of January 1966. Regular subscription rate: \$132.00 a year (6 Vol., 12 issues). Special rate: $\$ 66.00$ a year to individual members of supporting institutions.

Subscriptions, orders for numbers issued in the last three calendar years, and changes of address should be sent to Pacific Journal of Mathematics, P.O. Box 969, Carmel Valley, CA 93924, U.S.A. Old back numbers obtainable from Kraus Periodicals Co., Route 100, Millwood, NY 10546.

The Pacific Journal of Mathematics ISSN 0030-8730 is published monthly by the Pacific Journal of Mathematics at P.O. Box 969, Carmel Valley, CA 93924. Application to mail at Second-class postage rates is pending at Carmel Valley, California, and additional mailing offices. Postmaster: Send address changes to Pacific Journal of Mathematics, P. O. Box 969, Carmel Valley, CA 93924.

PUBLISHED BY PACIFIC JOURNAL OF MATHEMATICS, A NON-PROFIT CORPORATION

Copyright $(1984$ by Pacific Journal of Mathematics 


\section{Pacific Journal of Mathematics}

Vol. 110, No. $2 \quad$ October, 1984

Robert A. Bekes, The range of convolution operators $\ldots \ldots \ldots \ldots \ldots 257$

Dennis K. Burke and Sheldon Davis, Subsets of ${ }^{\omega} \omega$ and generalized metric

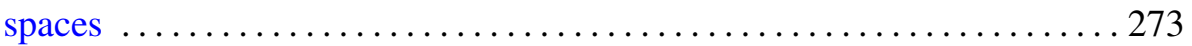

Giovanni Emmanuele, A remark on a paper: "Common fixed points of nonexpansive mappings by iteration" $\ldots \ldots \ldots \ldots \ldots \ldots \ldots \ldots \ldots 283$

I. Erdélyi and Sheng-Wang Wang, On strongly decomposable operators . . . 287

Gerhard Gierz, Injective Banach lattices with strong order units . . . . . . . 297

Maurizio Letizia, Quotients by complex conjugation of nonsingular quadrics and cubics in $\mathbf{P}_{\mathbf{C}}^{3}$ defined over $\mathbf{R} \ldots \ldots \ldots \ldots \ldots \ldots \ldots \ldots \ldots \ldots$

P. H. Maserick and Franciszek Hugon Szafraniec, Equivalent definitions

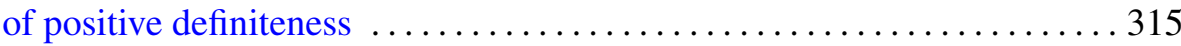

Costel Peligrad and S. Rubinstein, Maximal subalgebras of $C^{*}$-crossed

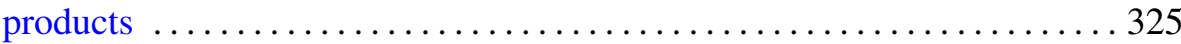

Derek W. Robinson and Sadayuki Yamamuro, Hereditary cones, order

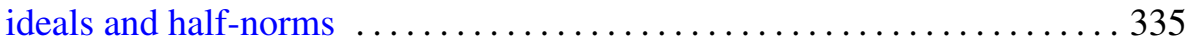

Derek W. Robinson and Sadayuki Yamamuro, The Jordan decomposition

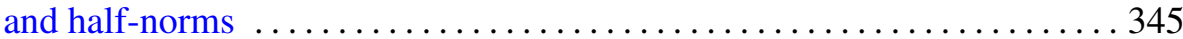

Richard Rochberg, Interpolation of Banach spaces and negatively curved

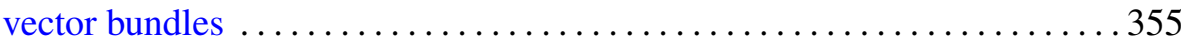

Dale Rolfsen, Rational surgery calculus: extension of Kirby's theorem 377

Walter Iaan Seaman, Helicoids of constant mean curvature and their Gauss maps

Diana Shelstad, Endoscopic groups and base change $\mathbf{C} / \mathbf{R}$

Jerrold Norman Siegel and Frank Williams, Numerical invariants of homotopies into spheres

Alladi Sitaram, Some remarks on measures on noncompact semisimple Lie groups

Teruhiko Soma, Atoroidal, irreducible 3-manifolds and 3-fold branched coverings of $S^{3}$

Jan de Vries, On the $G$-compactification of products

Hans Weber, Topological Boolean rings. Decomposition of finitely additive set functions 